\title{
.NET COMPILER USING CLOUD COMPUTING
}

\author{
Kavita M Garawad ${ }^{1}$, Girish $\mathbf{S}^{2}$ \\ ${ }^{1}$ Department of Computer Science and Engineering, Sahyadri College of Engineering and Management, Mangalore- \\ 575007 \\ ${ }^{2}$ Department of Computer Science and Engineering, Sahyadri College of Engineering and Management, Mangalore- \\ 575007
}

\begin{abstract}
Cloud computing has become a popular buzzword and it has been widely used to refer to different technologies, services and concepts. Compilers are used to run programs and convert them from a text format to executable format. Online .net compiler is the most convenient tool to compile the .net code and remove the errors. The errors/outputs of the code are stored in a more convenient way. And also avoids the trouble of installing the compiler on each computer. The main aim of the this paper is to provide the centralization compilation of the .net code with the help of internet connection and allows only authorized user can access their documents and applications from any computer over any Internet connection. this application helpful for conducting online examinations.
\end{abstract}

Keywords - Online Compiler, Cloud Computing

\section{INTRODUCTION}

Cloud computing is utility oriented approach and focuses on delivering services with a given pricing model; in most cases a pay per use strategy. Cloud computing allows anyone having a credit card to provision virtual hardware, runtime environments and services .The benefit from the use of cloud computing systems and technologies is the increased economical return due to reduced maintenance costs and operational costs related to infrastructure. As Cloud computing is a model for enabling convenient, on demand network access to a shared pool of configurable computing resources that can be rapidly provisioned and released with minimal management effort. We decided to make a project that aims to create an online compiler which helps to reduce the problems of portability of storage and space by making use of the concept of cloud computing. The use of compilers allows the programmer to pick up the fastest or convenient tool to compile the code and remove the errors. web based application can be used remotely through any network connection which is platform independent. The errors/Output of the compiled program can be stored in a more convenient way. Also the trouble of installing a compiler on each computer is avoided. this application helpful for conducting online examination.
$* * *$

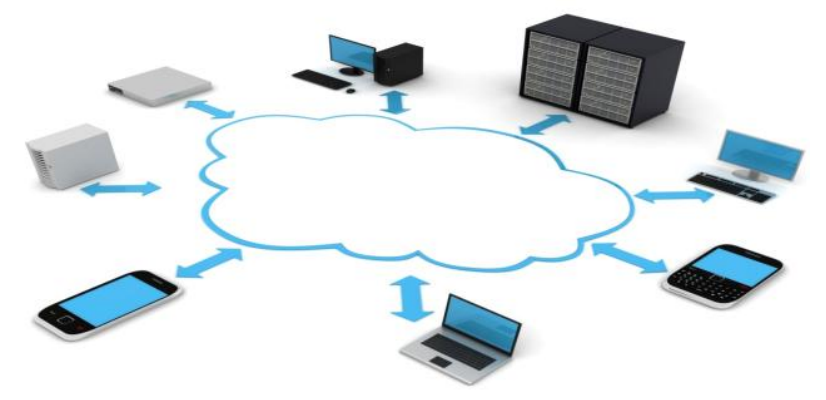

Fig 1.1: Cloud Computing

Three types of services provided by cloud

Saas (software as a service)

Paas (platform as a services)

Iaas (infrastructure as a service)

SaaS: Software as a Service (SaaS) tenders an application to user absolutely on On-Demand basis. Only one instance of the software runs on the cloud and the cloud caters to multiple users or clients i.e. users and applications [1]. Sass reduce IT operational costs by outsourcing software Hardware maintenance and support to the cloud provider. And also applications hosted in the server or centrally, updates can be released without the help of users to install new software. Disadvantage of saas is that user data stored on the cloud provider's server so data is not secure[2]. Software as a Service (SaaS) model, for security purpose client has to depend on the service provider. The provider must ensure that the users don't get to see each other's data. So it becomes important to the user to ensure that right security measures are important and also difficult to get an assurance of the application when needed [4]. 
While using SaaS model, the cloud customer will, substituting new software applications for old ones. Therefore, the focus on preserving the security functionality provided by the legacy application and achieving a successful data migration [3]

PaaS: Platform as a Service (PaaS) solution provide a development and deployment platform for running applications in the cloud That platform may encompass middleware, applications, OS and environment to develop those services. These platforms are really capable of handling user applications [1].

IaaS: Infrastructure as a Service (IaaS) includes necessities like storage, computing resources on the network. switches, servers, memory etc. and these are made available on network to IT architects to own whole infrastructure without actually installing all those physically [1].

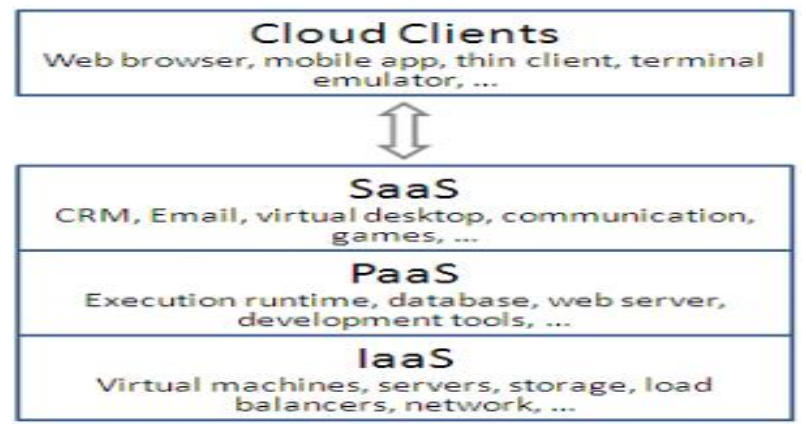

Fig 1.2: Cloud Computing Services

\section{NEED FOR PROJECT}

The main advantage of cloud computing over the other nonnetwork methods is of faster processing. Also, many processors can be used remotely, without the knowledge of the user, in order to expedite the processing. Thus, keeping this main advantage in mind, the main aim of the project the project is to provide a centralized compiling scheme for institutions. Also, it will act as a centralized repository for all the codes written. The other major advantage the others that it will make the users system lightweight i.e. there will be no need to maintain separate compilers/SDK's at the client-side. Thus, for educational institutions this will prove to be highly efficient. Also, the process of maintenance and distribution of dynamic usernames and passwords will be greatly simplified. Also, authentication and personalized task distribution will be made possible

\section{RELATED WORKS}

[1] Cloud computing implies a service oriented computing, utility oriented computing reduced information technology overhead, great flexibility, lower cost , and on demand

services among other advantages. The National Institute of Standards and Technology (NIST) defines _Cloud Computing refers to both the application delivered as services over the internet, and the hardware, system software in the datacenters provide those services. This definition describes cloud computing as phenomenon touching on the entire stack from the underlying hardware to the high level software services and applications.

[2] The concept of computing comes from grid, public computing and SaaS. This method that shares basic framework. Basic principles of cloud computing is to make the computing be assigned to number of computers, rather than local computer The concept of software as a service introduce a new delivery model for applications. It has been inherited from the of application service providers. These deliver software services based solutions across the wide area network from a central data centers and make them available on subscription on rental basis..This software delivery model is possible because economies of scale are reached by means of multy-tenancy.

[3] The characteristics of cloud computing are more complex. There are nineteen characteristics which can be used to distinguish cluster, grid and cloud computing systems. Grid computing proposed a new approach to access a large computational power, huge storage facilities, and a wide variety of services Grids initially developed as aggregation of geographically dispersed clusters by means of internet connection. These clusters belonged to different organizations and arrangements were made among them to share the computational power worldwide. Several reasons made possible the diffusion of computing grids: 1) Clusters were now resources quite common; 2) they were often under-utilized; 3) new problems were requiring computational power going beyond the capability of single clusters;4) the improvements in networking and the diffusion of internet made possible long distance high bandwidth connectivity. All these elements led to the development of grids, which now serve a multitude of users across the world. Clouds are characterized by the fact of having virtually infinite capacity, being tolerant to failures ,and always on as in the case of mainframes.

[4] Tow views of cloud computing, One is the cloud infrastructure other is cloud application. Infrastructures as a service solutions Provide the capabilities to add and remove resources. Application that leverages cloud computing benefit from its capability of dynamically scaling on demand

\section{DESIGN METHODOLOGY}

The primary functions of Centralized C\# Compiler using Cloud Computing are as fallows

Login Option: User can login with the user id and password

Compile option: Takes the code to the server for compilation of the code and compiler package has been imported.

Execute Option: In this option execution of the code takes place and results are displayed on the client window. 
The proposed system provides centralization compilation schema for the .net code using cloud computing .Software is hosted on the cloud and it is used as the service over the cloud. User first need to register and login with the user name and password after that user select the file and click on the compilation button, the code is submitted to the cloud for the authorised users otherwise it shows unsuccessful submission and code is encrypted during submission and decryption of the code takes place during receiving the code. After successful submission compilation of the code takes place in the cloud, exe file is generated and results are displayed on the client window if errors present in the code means error is displayed on the client window.

The main advantage of the proposed system is that it reduce storage space in the systems and also reduce the time needed to install the visual studio in the systems. Elegant and simple to use GUI for better coding. .Disadvantages of the proposed system are not cost effective and requirement of an internet connection.

\section{SNAPSHOTS}

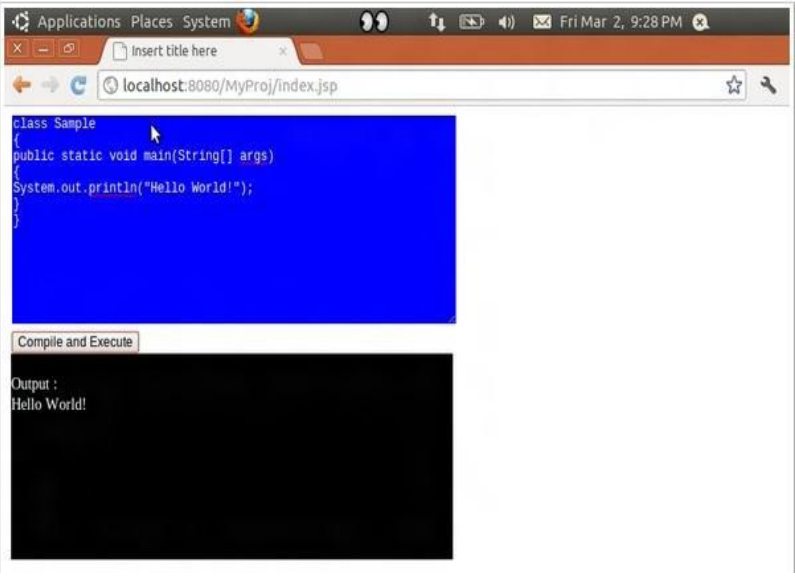

Fig 4.1 code is not having error

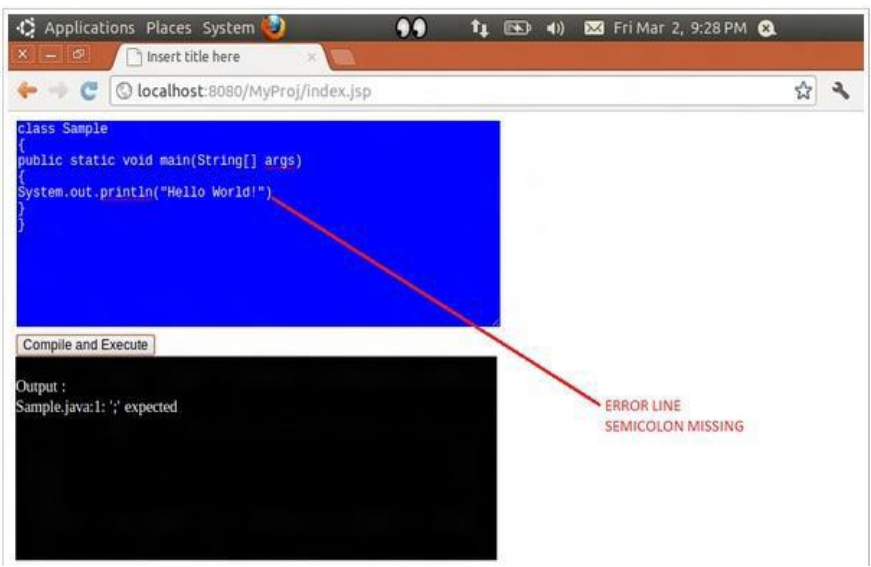

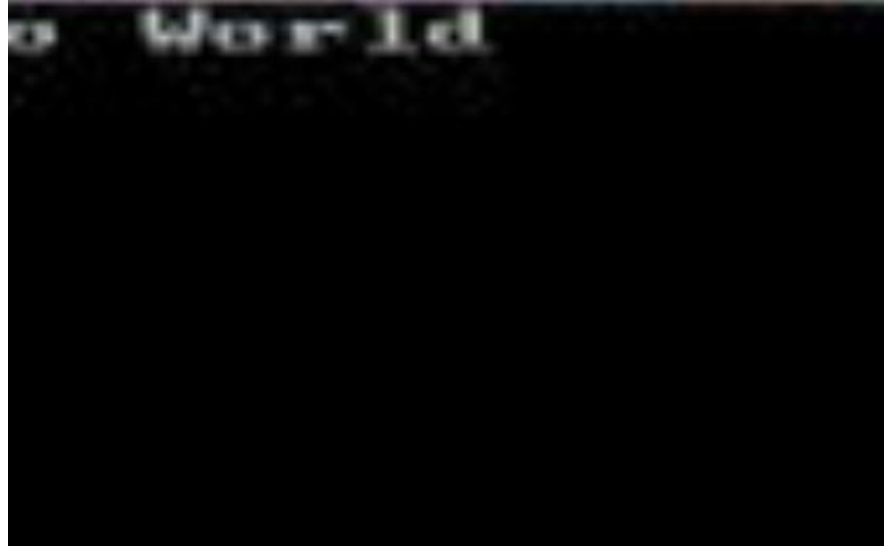

Output window

\section{CONCLUSIONS}

Implementing .NET Compiler using cloud computing reduce the problems of portability, storage and User don't have to install the huge and time consuming setup for visual studio. Project purpose is to provide provide compiler over network. Online compiler can be used in MNC for centralized database as well as centralized compiler installation. Due to centralized compiler no need of more configuration in the employee desktop. Online compiler can be used in school/college/Institutes for practical examination also for online examinations.

\section{REFERENCES}

[1]. http://www.cloudsherpas.com/resources/learn/saas-paasand-iaas/

[2]. http://en.wikipedia.org/wiki/Cloud_computing.

[3]. Seccombe A.., Hutton A, Meisel A, Windel A, Mohammed A, Licciardi A, (2009). Security guidance for critical areas of focus in cloud computing, v2.1 Cloud Security Alliance.

[4]. Choudhary V.(2007). Software as a service: implications for investment in software development. In: International conference on system sciences, 2007, p. 209.

Fig 4.2 code having error 